\title{
Psychotic-like experiences in esoterism: a twilight zone?
}

Barbara Hinterbuchinger ${ }^{1}$, Zsuzsa Litvan¹, Elias Laurin Meyer ${ }^{2}$, Fabian Friedrich ${ }^{1}$, Alexander Kaltenboeck ${ }^{3}$, Maria Gruber ${ }^{1}$, Daniel König ${ }^{1}$, Stefanie Sueßenbacher ${ }^{1}$, Nilufar Mossaheb ${ }^{1}$

${ }^{1}$ Department of Psychiatry and Psychotherapy, Clinical Division of Social Psychiatry, Medical University of Vienna, Austria ${ }^{2}$ Section for Medical Statistics, Center for Medical Statistics, Informatics, and Intelligent Systems, Medical University of Vienna, Austria

${ }^{3}$ Department of Psychiatry, University of Oxford, United Kingdom

Corresponding author:

Barbara Hinterbuchinger

Clinical Division of Social Psychiatry,

Department of Psychiatry and Psychotherapy,

Medical University of Vienna, Austria

Waehringer Guertel 18-20, 1090 Vienna, Austria

$\mathrm{Ph}:+43-1-4040035140$

e-mail: barbara.hinterbuchinger@meduniwien.ac.at

Word count: Abstract: 249

Text: 2479

Revised version for submission to: Schizophrenia Research 


\section{Abstract}

Background: Over the past decades, research has suggested the existence of a psychosis continuum ranging from psychotic-like experiences (PLEs) in the general population to psychotic symptoms in patients with affective and schizophrenia spectrum disorders. Especially individuals interested in esoterism were more often reported having experienced PLEs. However, there is little information on the extent of PLEs in this subculture. The aim of this study was to assess the extent of PLEs in a non-clinical population with interest in esoterism by means of an anonymized clinically used screening questionnaire.

Participants and Methods: The 16-item version of the Prodromal Questionnaire (PQ16), a self-report screening questionnaire assessing the presence of PLEs was administered to individuals with interest in esoterism (IE) and a control group without interest in esoterism (NI).

Results: The sample included 402 individuals. 224 subjects (55.7\%) reported interest in esoterism and 178 subjects (44.3\%) showed no such interest. In an ANCOVA, interest in esoterism was shown to have a significant impact on the PQ-16 score $(<0.001)$. Also, age $(p=0.022)$ and the interaction between age and interest in esoterism had a significant impact on the PQ-16 score ( $p=0.004)$. Specifically, younger individuals interested in esoterism showed increased PQ-16 scores, whereas scores decreased with increasing age. In individuals without interest in esoterism, age had no relevant impact on the score.

Conclusion: Younger individuals interested in esoterism seem to be more prone to reporting psychotic-like experiences compared to individuals without interest in esoterism and compared to their older counterparts.

Keywords: Psychotic-like experiences; Psychosis continuum; Esoterism;

\section{Introduction}

The last decades of research point towards a temporal and phenomenological continuum of psychotic experiences and suggest that psychotic symptoms occur not only in patients with schizophrenia-spectrum disorders (Morgan et al., 2009; Nuevo et al., 2012), but also in patients with affective and anxiety disorders (Hanssen et al., 2003) and individuals of the general, non-help-seeking population (Verdoux and van Os, 2002). 
Under the assumption of a psychosis continuum, the concept of the „at-risk mental state” (ARMS) has been described resulting in the development of operationalized criteria for the detection of individuals at ultra high risk (UHR) for developing full-blown psychosis (Yung et al., 2003). The UHR criteria include attenuated psychotic symptoms (APS), brief limited intermittent psychotic symptoms (BLIPS) or the genetic risk and deterioration (GRD) syndrome. In contrast to the ARMS concept, which is accompanied by distress and help-seeking behavior of the affected person, psychotic symptoms in the presumed absence of illness and distress are referred to as psychotic-like experiences (PLEs), also described as a non-clinical psychotic phenotype (Kelleher and Cannon, 2011). A metaanalysis by van Os et al. (Van Os et al., 2009) reported a median prevalence of 5-8\% for PLEs in the general population and even higher rates have been shown in children and adolescents (Laurens et al., 2007; Pontillo et al., 2016). Albeit subclinical, PLEs in children as well as in adults were shown to increase the risk of developing schizophrenia-spectrum disorders (Kelleher and Cannon, 2011; Poulton et al., 2000). This led to the idea of psychotic symptoms being distributed within different states of transition along a continuum, in different intensities and different stages of persistence, rather than perceiving them as a dichotomous feature, i.e. present or absent. The clinical meaning of PLEs within this transitory state is still indistinct today (Lee et al., 2016). Broadening the scope of research on this "twilight zone" of PLEs in different subgroups may provide new insights into the development of psychotic disorders, potential predictors and their aetiology.

PLEs are phenotypically similar to paranormal beliefs and experiences (Cella et al., 2012), which are common in the general population (Pechey and Halligan, 2011) and refer to "physically impossible" processes (Thalbourne, 1994) only explainable through the revision of scientific fundamentals (Tobacyk and Milford, 1983). Esoterism may be defined as practices involving the inner experiences of some form of faith, the mystical aspects, accessible only to the initiated; and on a broader, more colloquial level, the activities and faith involved around paranormal beliefs and experiences. Several studies have suggested a positive relationship between paranormal beliefs or experiences and PLEs (Lawrence and Peters, 2004; Thalbourne, 1994), schizophrenia-spectrum disorders (Peters et al., 2004) and schizotypy (Hergovich et al., 2008; Tobacyk and Wilkinson, 1990), including similar cognitive biases between paranormal and delusional beliefs (Blackmore and Trościanko, 1985; Lawrence and Peters, 2004; Wierzbicki, 
1985). Similar to the definition of the "non-clinical psychotic phenotype", MyCreery and Claridge described the concept of the "healthy schizotype" as referring to an individual's perceptual anomalies and out-of-body-experiences without impairment of cognition or social functioning (McCreery and Claridge, 2002).

Recent research has shown interest in PLEs in individuals of special subpopulations. Powers et al. examined non-help-seeking clairaudient psychics receiving daily auditory messages in comparison with psychotic patients with acoustic hallucinations. Interestingly, the psychics with acoustic hallucinations without any help-seekingbehavior were able to control the onset and offset of their voices, and were less distressed and received a more positive reaction when admitting to voice-hearing for the first time (Powers et al., 2016).

Since the clinical role of PLEs is still a matter of research, the aim of this study was to investigate the prevalence of PLEs in a non-clinical general population sample of individuals with interest in esoterism compared to a sample of individuals without any interest in esoterism. To the authors' knowledge, this is the first study assessing the presence of PLEs in a subpopulation in which PLEs might be more common, those interested in esoterism, by means of a clinically used screening instrument originally developed to identify at-risk mental state individuals. Since PLEs have been reported as occurring significantly more often at younger age (Laurens et al., 2007; Pontillo et al., 2016), we also assessed the relationship between the extent of PLEs in each group and age. In this study, esoterism and paranormal beliefs/experiences are used with the broader definition above, i.e. as an umbrella term referring to the manifold field of subjects including clairvoyance, mediumship and channeling etc.

\section{Methods}

\subsection{Sample}

The study sample is a non-help-seeking general population sample of 224 individuals with (IE) and 178 individuals without interest (NE) in esoterism. Women and men aged 18 to 90 years were eligible to participate. 


\subsection{Procedure}

The anonymized 16-item version of the Prodromal Questionnaire (PQ-16) (Ising et al., 2012) was administered to a non-clinical general population sample of individuals with interest in esoterism as well as a control group of individuals without interest in esoterism during a large esoterism fair in October of 2015 in Vienna, Austria. The esoterism fair took place at a large event venue at a busy and well-frequented location in Vienna. Individuals were approached by members of the study team and asked whether they were attending the esoterism fair and had special interest in esoterism and whether they were willing to fill out our questionnaire. They were given a questionnaire consisting of the16-item Version of the Prodromal Questionnaire as well as three additional questions regarding sex, age and interest in esoterism (yes/no). No further information was given addressing the study. Recruitment of further study participants was performed via concentric-circle-recruitment. The research protocol was approved by the local ethics committee.

\subsection{Assessments}

\subsubsection{The 16-Item Version of the Prodromal Questionnaire (PQ-16)}

The 16-item Version of the Prodromal Questionnaire is a self-report screening questionnaire developed on the basis of the Prodromal Questionnaire (PQ) (Loewy et al., 2005) and aims to identify individuals with high risk for psychosis for a further in-depth diagnostic interview (Ising et al., 2012). It assesses the presence of psychotic-like experiences on a two-point scale (true/false) and includes nine items assessing perceptual abnormalities and hallucinations, five items regarding unusual thought content, delusional ideas and paranoia and two items assessing negative symptoms. The PQ-16 questionnaire has been tested as a diagnostic screening instrument for the attenuated psychosis syndrome in secondary mental health care with satisfactory performance (Ising et al., 2012).

\subsection{Statistics}

Descriptive statistics of all variables of interest were compiled. An ANCOVA was used to explain the PQ-16 score by interest in esoterism, age and sex, as well as their respective interactions. Furthermore, logistic regressions were used to explain each single outcome of the 16 questions of the PQ-16 questionnaire by the interest in esoterism, age 
and sex. P-values from the logistic regressions serve only descriptive purposes; hence no multiple testing corrections were applied. All statistical analysis was conducted using the Statistical Package for Social Sciences (SPSS), Version 23.0 and the Statistic Software R 3.2.5

\section{Results}

The final sample included 402 individuals. Participants were between 19 and 83 years old. Of the final sample, 224 subjects (55.7\%) reported interest in esoterism and 178 subjects (44.3\%) showed no interest in esoterism. There were more female subjects in the total sample (71.4\%). In the IE group there were more women (58.2\% female subjects) compared to the NI group (41.8\% female subjects). The mean PQ-16 score of IE individuals $(6.24 \pm 3.47)$ was 4.04 points higher compared to NI individuals $(2.20 \pm$ 2.48) (p-value < 0.001) (see Tab.1).

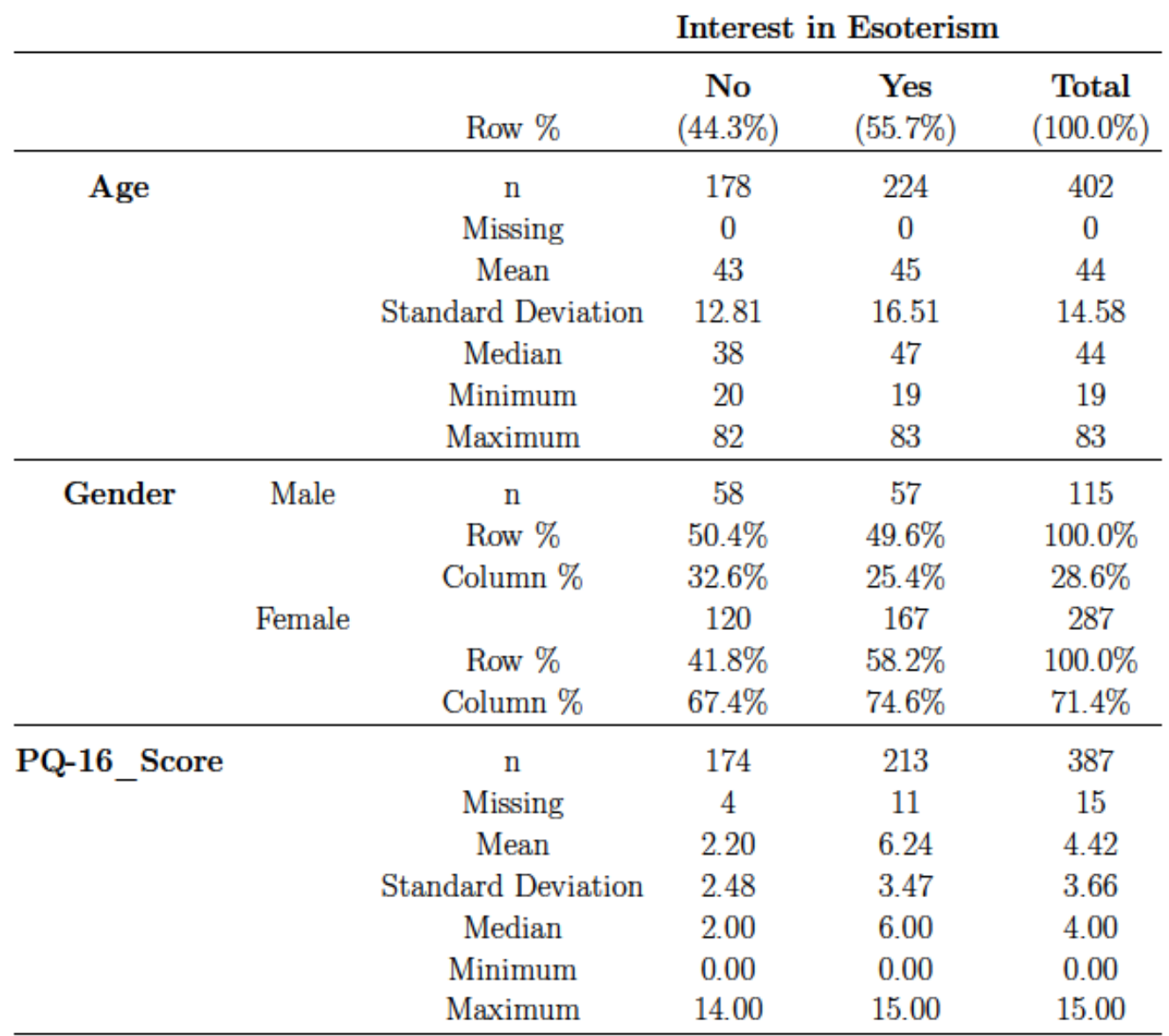

Tab. 1: Descriptive statistics of age, gender and PQ-16 score $\mathrm{w} / \mathrm{r}$ to interest in esoterism 
An ANCOVA was performed with PQ-16 score as the dependent variable and interest in esoterism, age, sex and the interaction between interest in esoterism and age/sex as independent variables. Interest in esoterism showed a significant impact on the PQ-16 score $(<0.001)$. Also, age $(p=0.022)$ and the interaction between age and interest in esoterism had a significant impact on the PQ-16 score ( $p=0.004)$.

While the expected PQ-16 score of IE individuals decreases with increasing age, a minimal increase in the PQ-16 score (0.002 per year) could be shown for NI individuals (see Fig.1).

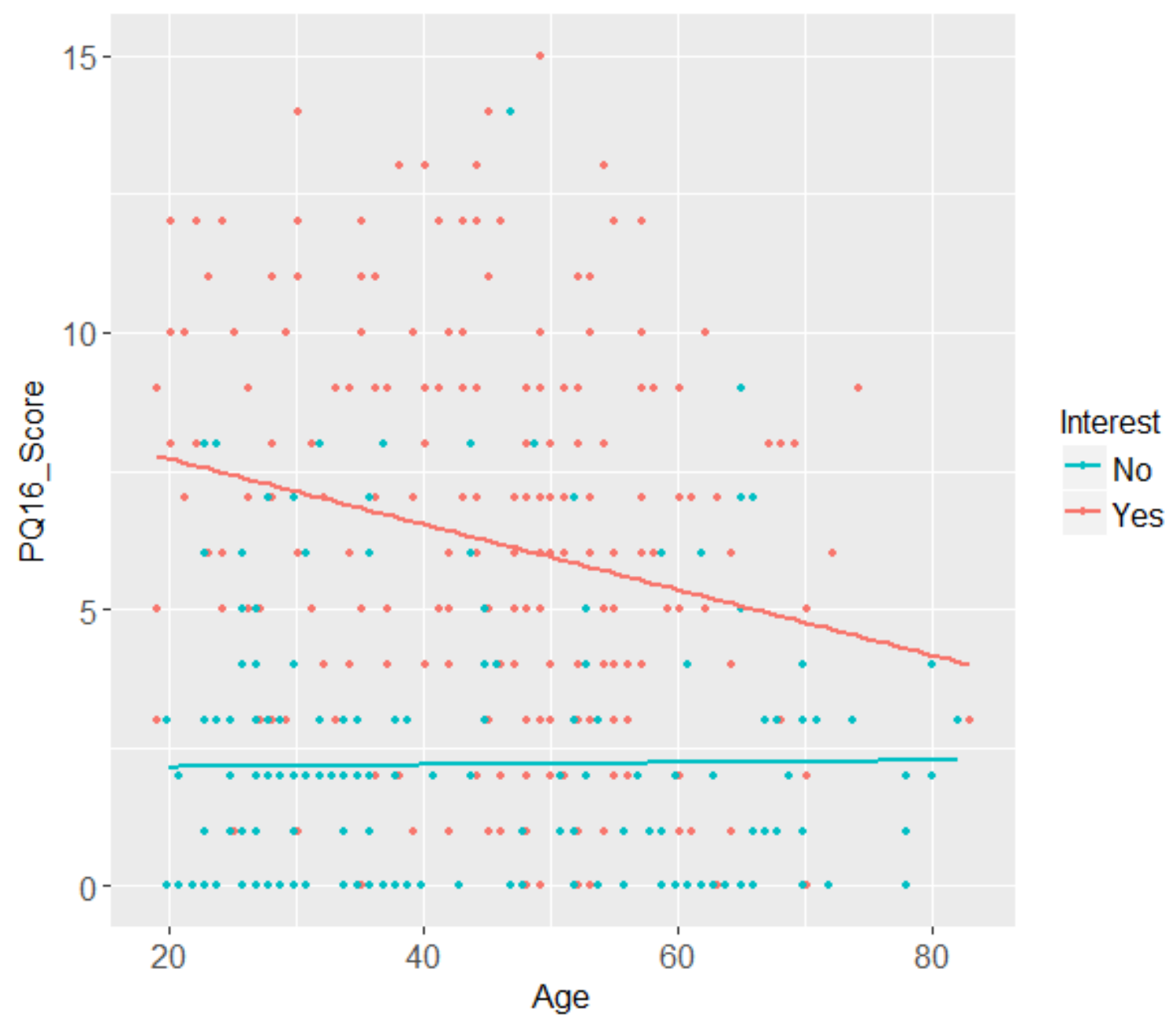

Fig.1: Regression lines $\mathrm{w} / \mathrm{r}$ to interest in esoterism

Sex had no significant impact on the PQ-16 score and no significant difference could be shown in the mean PQ-16 score regarding sex ( $p=0.697)$ (see Fig.2). 


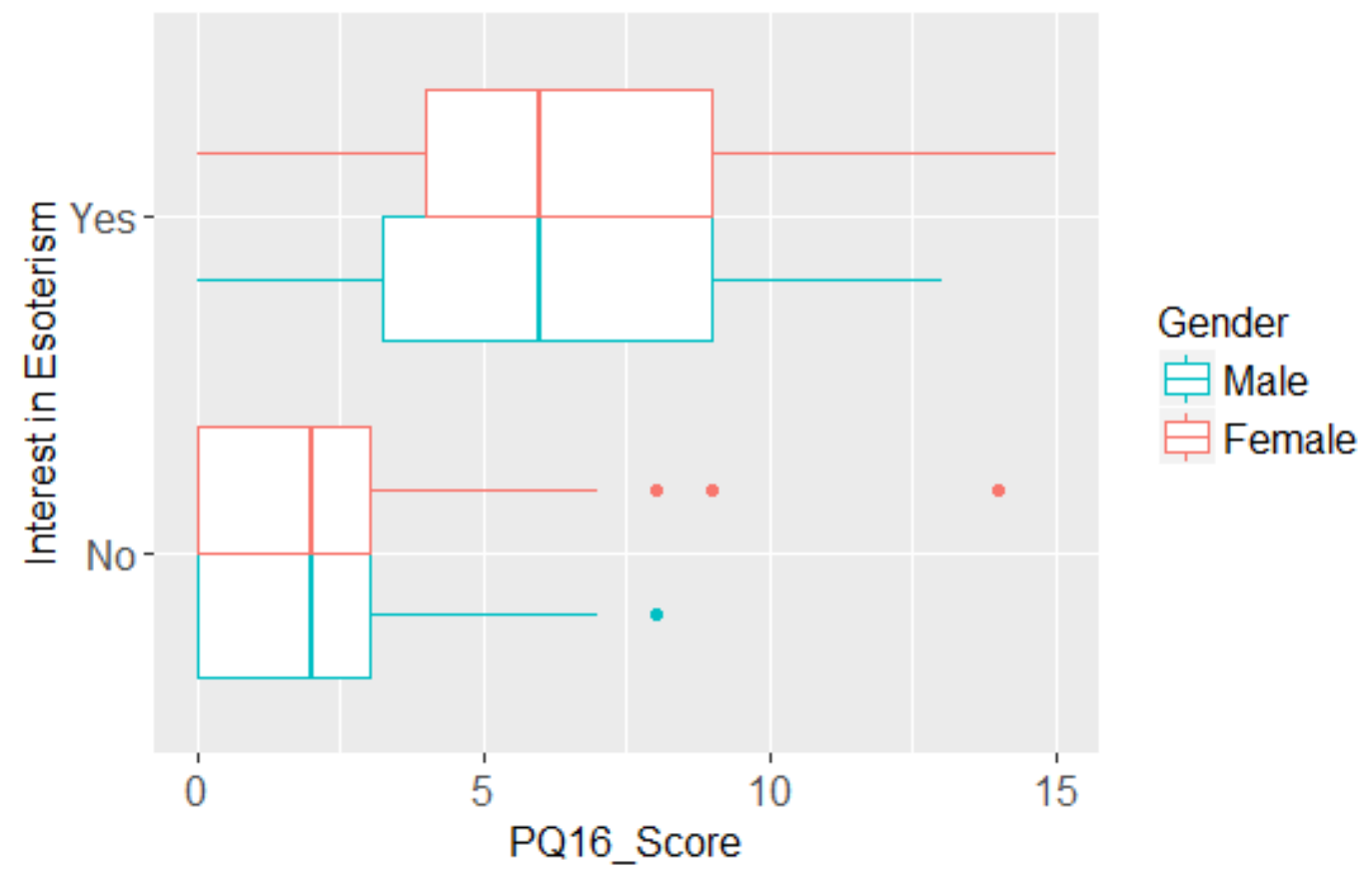

Fig.2: PQ16 - Score $\mathrm{w} / \mathrm{r}$ to sex and interest in esoterism

Individuals with interest in esoterism had significantly more positive answers in the PQ16 questionnaire. With exception of question 7 ("I get extremely anxious, when I meet people for the first time."; $\mathrm{p}=0.024$ ), results would still be significant after Bonferroni correction (significant if $\mathrm{p}<0.05 / 16=0.003125$ ). Age had a significant impact on participants' answers for the following questions: Question 4 ("I often hear anomalous sounds like knocking, clicking, hissing, clapping or ringing."; $p=0.022$ ), question 5 ("Sometimes I feel confused, if something I experience is real or imagined."; $p<0.001$ ), question 7 ("I get extremely anxious, when I meet people for the first time."; p=0.001), question 9 ("Sometimes my thoughts are so strong that I can almost hear them."; $\mathrm{p}=0.032$ ) and question 11 ("Sometimes I have the feeling, that I don't have control over my feelings and thoughts."; $p=0.017$ ), showing that younger age was associated with more affirmative answers. In 3 of the questions, sex had a significant impact on participants' answers: More men than women answered „yes“ to question 1 ("I have lost interest in things I enjoyed in the past."; p=0.013) and question 16 ("I had the feeling that parts of my body have changed or work in a different way than before."; $p=0.008$ ). More women compared to men responded affirmatively on question 15 ("I had the feeling that a person or energy was around me, although I did not see anything.") $(p=0.009)$ (see Tab.2). 


\begin{tabular}{cccc}
\hline \hline & Interest in Esoterism & Age & Gender \\
\hline Q1 & $<0.001$ & 0.716 & 0.013 \\
Q2 & $<0.001$ & 0.855 & 0.314 \\
Q3 & $<0.001$ & 0.528 & 0.948 \\
Q4 & $<0.001$ & 0.022 & 0.258 \\
Q5 & $<0.001$ & $<0.001$ & 0.537 \\
Q6 & $<0.001$ & 0.530 & 0.102 \\
Q7 & 0.024 & 0.001 & 1 \\
Q8 & $<0.001$ & 0.651 & 0.621 \\
Q9 & $<0.001$ & 0.032 & 0.990 \\
Q10 & $<0.001$ & 0.154 & 0.126 \\
Q11 & $<0.001$ & 0.017 & 0.762 \\
Q12 & 0.001 & 0.086 & 0.897 \\
Q13 & $<0.001$ & 0.704 & 0.524 \\
Q14 & $<0.001$ & 0.158 & 0.287 \\
Q15 & $<0.001$ & 0.378 & 0.009 \\
Q16 & $<0.001$ & 0.749 & 0.008 \\
\hline
\end{tabular}

Tab.2: P-values of univariate logistic regressions

\section{Discussion}

In this study, a non-clinical sample of individuals interested in esoterism showed significantly higher scores on an anonymized self-report screening instrument for psychotic-like experiences compared to individuals without interest in esoterism.

Higher scores in the mean PQ-16 score were shown especially in the younger individuals interested in esoterism. While the mean PQ-16 score decreased with increasing age in the group of individuals interested in esoterism, the score remained almost unchanged with respect to age in the control group. In the general help-seeking population, a cutoff score of 6 or more positively answered items on the PQ - 16 produced correct classification of psychosis risk or clinical psychosis in $44 \%$ of the cases, distinguishing individuals at ultra high risk (UHR) from individuals with no UHR state with high sensitivity and specificity (both 87\%) (Ising et al., 2012). Since we examined a nonclinical sample of the general population, no conclusions can be drawn if and to which extent individuals at UHR are part of this subpopulation, although it should be mentioned that individuals interested in esoterism showed a mean PQ-16 score above the clinically used cut-off. Considering that individuals at UHR may possibly hide their symptoms under the curtain of esoterism, the question arises whether interest in 
esoterism or paranormal beliefs should be considered during the assessment of UHR states and in how far the operationalized criteria for UHR could benefit from this consideration. Given the fact that many psychiatric disorders, and specifically psychotic disorders, typically become manifest during adolescence or young adulthood, the question emerges whether some individuals at high-risk of developing a psychotic disorder or even already experiencing a psychotic episode might look for answers or explanations in a special subculture in which anomalous beliefs and unusual experiences are common or even desired. The association of younger age with higher PQ-16 scores in the IE group could be interpreted as mirroring general prevalence rates of PLEs of up to $48 \%$ in adolescents (Chambers et al., 1982). However, we did find such an age association only in those interested in esoterism, and not in the control group, thus emphasizing the effect of interest in esoterism itself. Indeed, belief in esoterism seems to be more common in adolescents than in older individuals, with traditional religious beliefs as an exception (Irwin, 1993). This might reflect on young adulthood as a time of transition, of coming-of-age, with increased preoccupation with the quest for meaning in life, questioning of value systems, personal life planning and subsequently emerging difficulties, which might result in a search for answers and support in various subcultural topics, such as esoterism. The question arises whether occupation with esoterism could present an additional stressor in psychosis-prone individuals, thereby facilitating the development of psychosis. Possibly, in special subcultural groups such as those of esoterism, PLEs might not only be experienced more often, but even be desired, increasing the probability of socially desired experiences or just answers. This is the first study of its kind showing significantly higher levels of PLEs in people interested in esoterism, and even more so, in younger IE individuals having higher scores.

However, our study also bears some limitations. In this sample, no inferences can be made about UHR states, blunt psychotic symptoms or psychotic disorders, nor about any direction of causality. Furthermore, the PQ-16 questionnaire is not sensitive enough to distinguish between UHR syndromes and psychosis. For an accurate examination of atrisk mental states, further in-depth clinical interviews with standardized instruments would have been necessary. This was not done in this sample because an anonymized version of the questionnaire was used aiming to avoid major social desirability biases, as well as refusal to participate due to fear of stigmatization with a psychiatric diagnosis. Our sample is not a specifically selected sample and we used "interested in esoterism" 
vs. "not interested in esoterism" as the only selection factor. Therefore, we avoided a selection bias that may be found in studies with narrower inclusion criteria and those performed in clinical settings. Furthermore, we can safely assume that our sample included a wide range of different variations of „interest in esoterism“, making our highly significant results even more interesting. The results cannot be generalized to other populations. Our study, which clearly shows a positive relationship between PLEs and interest in esoterism, leaves room for interpretation of direction of causality in future studies.

Finally, in line with the idea of a psychosis continuum, a clear distinction between PLEs, pathological perceptual aberrations and special beliefs and experiences within subcultural norms can often be hard to draw. Since hallucinatory-like experiences seem to be much more prevalent in the general population than their clinical manifestations (Van Os et al., 2009), caution should be applied regarding possible over-pathologization of anomalous or psychotic-like experiences in non-help seeking individuals. Future research focusing on PLEs in special subgroups might bring further knowledge and new perspectives on this "twilight zone" by addressing the inconclusive meaning of PLEs in the general population. Moreover, addressing psychotic-like experiences in homogenous sub-populations may help to identify protective factors undetected in more heterogeneous samples.

\section{Role of funding source}

No funding.

\section{Contributors}

Author BH contributed to: design of the study, literature searches, data acquisition, interpretation of the results and drafted the manuscript.

Author NM contributed to: design and implementation of the study, supervision, analysis, interpretation of results and writing of the manuscript.

Authors ZL, SS, DK, MG contributed to: data acquisition and writing of the manuscript. Authors FF and AK contributed to: design of the study, interpretation of results and writing of the manuscript.

\section{Conflict of interest}


AK receives funding from the U.K. Medical Research Council. The other authors declare that they have no conflicts of interest.

\section{Acknowledgement}

We would like to thank Benjamin Missbach for his support at data acquisition and Ingrid Salazar for editing of the manuscript.

\section{References}

Blackmore, S., Trościanko, T., 1985. Belief in the paranormal: Probability judgements, illusory control, and the 'chance baseline shift'. British journal of psychology 76(4), 459468.

Cella, M., Vellante, M., Preti, A., 2012. How psychotic-like are paranormal beliefs? J Behav Ther Exp Psychiatry 43(3), 897-900.

Chambers, W.J., Puig-Antich, J., Tabrizi, M.A., Davies, M., 1982. Psychotic symptoms in prepubertal major depressive disorder. Archives of general psychiatry 39(8), 921-927. Hanssen, M., Peeters, F., Krabbendam, L., Radstake, S., Verdoux, H., van Os, J., 2003. How psychotic are individuals with non-psychotic disorders? Social psychiatry and psychiatric epidemiology 38(3), 149-154.

Hergovich, A., Schott, R., Arendasy, M., 2008. On the relationship between paranormal belief and schizotypy among adolescents. Personality and Individual Differences 45(2), 119-125.

Irwin, H.J., 1993. Belief in the paranormal: A review of the empirical literature. Journal of the american society for Psychical research 87(1), 1-39.

Ising, H.K., Veling, W., Loewy, R.L., Rietveld, M.W., Rietdijk, J., Dragt, S., Klaassen, R.M., Nieman, D.H., Wunderink, L., Linszen, D.H., van der Gaag, M., 2012. The validity of the 16item version of the Prodromal Questionnaire (PQ-16) to screen for ultra high risk of developing psychosis in the general help-seeking population. Schizophr Bull 38(6), 1288-1296.

Kelleher, I., Cannon, M., 2011. Psychotic-like experiences in the general population: characterizing a high-risk group for psychosis. Psychological medicine 41(1), 1-6. Laurens, K.R., Hodgins, S., Maughan, B., Murray, R.M., Rutter, M.L., Taylor, E.A., 2007. Community screening for psychotic-like experiences and other putative antecedents of schizophrenia in children aged 9-12 years. Schizophrenia research 90(1), 130-146. Lawrence, E., Peters, E., 2004. Reasoning in believers in the paranormal. J Nerv Ment Dis 192(11), 727-733.

Lee, K.W., Chan, K.W., Chang, W.C., Lee, E.H., Hui, C.L., Chen, E.Y., 2016. A systematic review on definitions and assessments of psychotic-like experiences. Early Interv Psychiatry 10(1), 3-16.

Loewy, R.L., Bearden, C.E., Johnson, J.K., Raine, A., Cannon, T.D., 2005. The prodromal questionnaire (PQ): preliminary validation of a self-report screening measure for prodromal and psychotic syndromes. Schizophrenia research 79(1), 117-125. McCreery, C., Claridge, G., 2002. Healthy schizotypy: The case of out-of-the-body experiences. Personality and Individual Differences 32(1), 141-154.

Morgan, C., Fisher, H., Hutchinson, G., Kirkbride, J., Craig, T.K., Morgan, K., Dazzan, P., Boydell, J., Doody, G.A., Jones, P.B., Murray, R.M., Leff, J., Fearon, P., 2009. Ethnicity, social 
disadvantage and psychotic-like experiences in a healthy population based sample. Acta Psychiatr Scand 119(3), 226-235.

Nuevo, R., Chatterji, S., Verdes, E., Naidoo, N., Arango, C., Ayuso-Mateos, J.L., 2012. The continuum of psychotic symptoms in the general population: a cross-national study. Schizophr Bull 38(3), 475-485.

Pechey, R., Halligan, P., 2011. The prevalence of delusion-like beliefs relative to sociocultural beliefs in the general population. Psychopathology 44(2), 106-115.

Peters, E., Joseph, S., Day, S., Garety, P., 2004. Measuring delusional ideation: the 21-item Peters et al. Delusions Inventory (PDI). Schizophr Bull 30(4), 1005-1022.

Pontillo, M., De Luca, M., Pucciarini, M.L., Vicari, S., Armando, M., 2016. All that glitters is not gold: prevalence and relevance of psychotic-like experiences in clinical sample of children and adolescents aged 8-17 years old. Early Interv Psychiatry.

Poulton, R., Caspi, A., Moffitt, T.E., Cannon, M., Murray, R., Harrington, H., 2000.

Children's self-reported psychotic symptoms and adult schizophreniform disorder: a 15year longitudinal study. Archives of general psychiatry 57(11), 1053-1058.

Powers, A.R., Kelley, M.S., Corlett, P.R., 2016. Varieties of voice-hearing: psychics and the psychosis continuum. Schizophrenia Bulletin, sbw133.

Thalbourne, M.A., 1994. Belief in the paranormal and its relationship to schizophrenia relevant measures: A confirmatory study. British Journal of Clinical Psychology 33(1), 78-80.

Tobacyk, J., Milford, G., 1983. Belief in paranormal phenomena: Assessment instrument development and implications for personality functioning. Journal of personality and social psychology 44(5), 1029.

Tobacyk, J.J., Wilkinson, L.V., 1990. Magical thinking and paranormal beliefs. Journal of Social Behavior and Personality 5(4), 255.

Van Os, J., Linscott, R.J., Myin-Germeys, I., Delespaul, P., Krabbendam, L., 2009. A systematic review and meta-analysis of the psychosis continuum: evidence for a psychosis proneness-persistence-impairment model of psychotic disorder.

Psychological medicine 39(02), 179-195.

Verdoux, H., van Os, J., 2002. Psychotic symptoms in non-clinical populations and the continuum of psychosis. Schizophrenia research 54(1-2), 59-65.

Wierzbicki, M., 1985. Reasoning Errors and Belief in the Paranormal. The Journal of social psychology 125(4), 489-494.

Yung, A.R., Phillips, L.J., Yuen, H.P., Francey, S.M., McFarlane, C.A., Hallgren, M., McGorry, P.D., 2003. Psychosis prediction: 12-month follow up of a high-risk ("prodromal") group. Schizophrenia research 60(1), 21-32. 